\title{
Iatrogenic Takotsubo Cardiomyopathy after Accidental Injection of Adrenaline-A Case Report
}

\author{
Mirela Ionescu $^{*}$, Louise Vallet ${ }^{2}$, François Milesi' ${ }^{1}$, Martine Ferro ${ }^{1}$, Younes Ait Tamlihat ${ }^{1,3}$ \\ ${ }^{1}$ Departement d'anesthésie, centre hospitalier de Saintonge 11 Boulevard Ambroise Paré 17100 Saintes, France
}

${ }^{2}$ Interne du centre hospitalier universitaire de Poitiers, 2 Rue de la Milétrie, 86021 Poitiers, France

${ }^{3}$ Service de réanimation polyvalente et soins continus, centre hospitalier de Saintonge 11 Boulevard Ambroise Paré 17100 Saintes, France

*Corresponding author: Mirela Ionescu, Departement d'anesthésie, centre hospitalier de Saintonge 11 Boulevard Ambroise Paré 17100 Saintes, France.

Citation: Ionescu M, Vallet L, Milesi F, Ferro M, Ait Tamlihat Y (2021) Iatrogenic Takotsubo Cardiomyopathy after Accidental Injection of Adrenaline-A Case Report. Ann Case Report 6: 717. DOI: 10.29011/2574-7754.100717

Received Date: 20 December 2021; Accepted Date: 24 December, 2021; Published Date: 29 December 2021

\begin{abstract}
Takotsubo cardiomyopathy is characterized by a sudden contractile dysfunction, generally transient but can be followed by rare complications and recurrence. Drug-related occurrence is described in the literature, associated with catecholamines. We report a case of Takotsubo syndrome after accidental injection of adrenaline instead of neostigmine in the context of a decurarization in the postanesthesia room, complicated by cardiorespiratory arrest. The immediate evolution was marked in post-extubation by the introduction of vasopressors in the face of hypotension and the discovery of an iatrogenic Takotsubo with collapsed LVEF and a ballooned aspect of the apex. The patient was discharged from the hospital after 13 days of hospitalization.
\end{abstract}

Keywords: Cardiomyopathy; Tako-Tsubo; Medication error; Epinephrine; Echocardiography; Apical balloon syndrome

\section{Introduction}

Takotsubo cardiomyopathy (TTC) or stress cardiomyopathy, also known as "broken heart syndrome" or "transient ballooning of the left ventricle" is an acquired syndrome associating a transient contractile dysfunction with a ballooned appearance of the apex. It was first described by Sato in 1990 where the term Takotsubo "octopus trap" in Japanese was used as a reference for the particular shape of the left ventricular cavity [1]. Although TTC is a reversible condition, the acute phase exposes patients to numerous complications and recurrence [2]. The direct toxicity of catecholamines on cardiomyocytes is one of the pathophysiological hypotheses of this syndrome either by massive endogenous secretion as in certain clinical contexts (pheochromocytoma, subarachnoid hemorrhage etc) or by important exogenous contribution of catecholamines which can be by drug administration error. We report a case of iatrogenic TTC after accidental injection of adrenaline.

\section{Case Description}

A 61-year-old woman with no cardiovascular risk factors and a main history of high-grade serous endometrial carcinoma discovered 3 years priorly and initially treated by surgery, radiotherapy and brachytherapy, then by several lines of chemotherapy for a year and a half, before having a relapse in the form of peritoneal carcinosis responsible for significant general deterioration due to a chronic subocclusive syndrome. A fibroscopy with implantation of a duodenal prosthesis was scheduled in the operating room. The patient was classified as ASA 3 and a transthoracic echocardiography (TTE) evaluation 4 months earlier showed a normal left ventricular ejection fraction (LVEF). The procedure was performed under general anesthesia with tracheal intubation and maintenance anesthesia with sevoflurane, sufentanil and atractrium. The procedure was carried out without intraoperative complications and the patient left intubated and ventilated in the postanesthesia room (PAR), being under a deep curarization. 
Citation: Ionescu M, Vallet L, Milesi F, Ferro M, Ait Tamlihat Y (2021) Iatrogenic Takotsubo Cardiomyopathy after Accidental Injection of Adrenaline-A Case Report. Ann Case Report 6: 717. DOI: 10.29011/2574-7754.100717

In the PAR, once the train of four (TOF) showed 4 responses out of 4 , a decurarization was performed by the anesthesia nurse (AN) after agreement of the anesthesiologist with $40 \mathrm{mcg} / \mathrm{kg}$ of prostigmine and $20 \mathrm{mcg} / \mathrm{kg}$ of atropine. The AN had injected $2.4 \mathrm{mg}$ of adrenaline instead of prostigmine plus $1 \mathrm{mg}$ of atropine. The patient became very tachycardic and the AN quickly realized her mistake because she had not yet discarded the ampoules. When the anesthesiologist arrived, the patient was in cardiorespiratory arrest due to ventricular fibrillation (Figure1), external cardiac massage was started and 3 external electric shocks were delivered, allowing a resumption of spontaneous cardiac activity after $5 \mathrm{~min}$ of low cardiac output. Given the stability of the clinical condition, the patient was extubated in the PAR and informed about the drug error. A few minutes later, a very labile systolic blood pressure was noted, justifying the initiation of noradrenaline after moderate filling with crystalloids. Emergency blood workup showed troponins below $10 \mathrm{ng} / \mathrm{L}$ and $\mathrm{BNP}$ at $43 \mathrm{pg} / \mathrm{mL}$. The on-call cardiologist performed a TTE showing a low LVEF estimated at $20 \%$, akinesia of the apex of the left ventricle with dilated aspect of the apex, compatible with TTC. Basal kinetics remained preserved, left atrium and right chambers not dilated. No pulmonary hypertension was observed, the pericardium had no fluid and the inferior vena cava was flat.

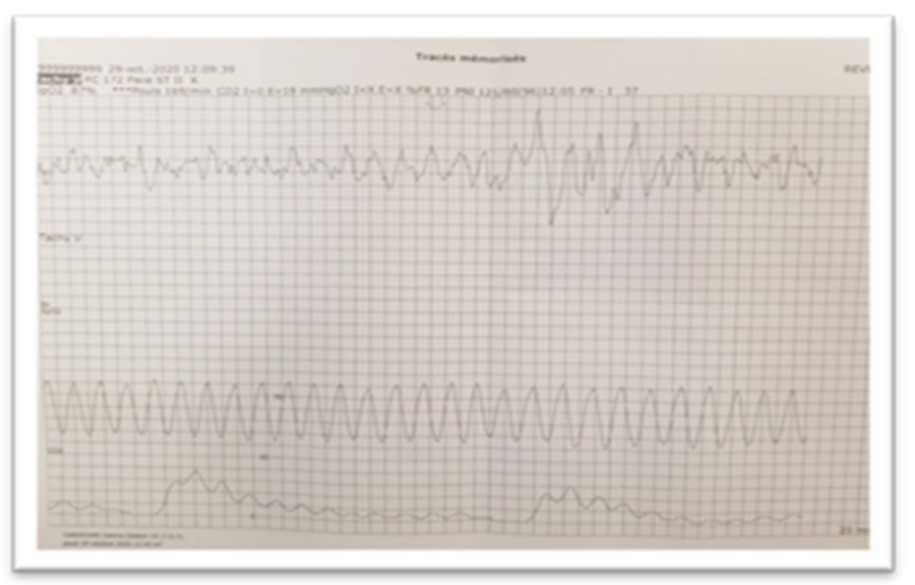

Figure 1: Electrocardiogram at initial management showing a pattern of ventricular fibrillation.

The patient was transferred to the cardiac intensive care unit and put on dobutamine at $10 \mathrm{mcg} / \mathrm{kg} / \mathrm{min}$ and noradrenaline was increased to $2 \mathrm{mg} / \mathrm{h}$. The subsequent evolution was marked by an episode of atrial fibrillation that resolved after the infusion of amiodarone, a troponin peak of $2600 \mathrm{ng} / \mathrm{L}$ at day 1 with rapid decrease thereafter. No ST-segment changes on the electrocardiogram (ECG) and daily TTE monitoring showed the absence of apical thrombus and beginning of LVEF recovery from day 6 allowing definitive weaning of the amines. The patient was transferred to the oncology department at day 7 and left the hospital after 13 days of hospitalization.

\section{Discussion}

TTC is an acquired and reversible cardiomyopathy affecting more women than men. According to published literature data, approximately $90 \%$ of patients with TTC are women with an average age of $67-70$ years $[3,4]$. It is characterized by left ventricular dysfunction without significant coronary artery disease, accompanied by akinesia involving apical segments in the typical form or mid-ventricular in the atypical forms [5]. In its clinical phenotype, chest pain, dyspnea as well as abrupt elevation of troponins and sometimes even ECG changes are often present, making the diagnosis difficult in the face of a strong suspicion of acute coronary syndrome (ACS). In this context, coronary angiography with left ventriculography allows to confirm the diagnosis. In our case, this examination was not performed and the diagnosis was sustained due to the context of iatrogeny, the TTE evaluation and the postoperative evolution. There are currently several diagnostic criteria for TTC. However, it is currently recognized by several experts that the presence of obstructive coronary artery disease should not systematically exclude TTC for several reasons: ACS can be a trigger for TTC, the coexistence of the two syndromes is possible and finally in some patients misdiagnosed as ACS the wall motion abnormalities usually extend beyond the territory of the coronary artery involved [6].

Post Takotsubo cardiomyopathy complications are: congestive heart failure, pulmonary edema and cardiogenic shock. It is proven that patients with cardiogenic shock have a higher mortality [7]. These at-risk patients often require ventilatory support, advanced cardiovascular support and cardiopulmonary resuscitation. The use of ECMO for cardiogenic shock associated with TTC has better prognosis [8].

Although the pathophysiology of TTC is not yet fixed, nevertheless, increased secretion of catecholamines following sympathetic hyperstimulation is often suggested. Thus, the mechanism by which catecholamines can induce left systolic dysfunction with ballooning of a myocardial segment is not yet clear. Several hypotheses have been proposed such as: plaque rupture, microcirculatory dysfunction, coronary microvessel spasm and direct catecholamine toxicity [5,6]. Adrenaline is one of the sympathomimetic drugs that can precipitate or trigger TTC and many observations are published in the literature where adrenaline is identified as a drug responsible for TTC either because of the use of high doses or by administration error [5]. 
Citation: Ionescu M, Vallet L, Milesi F, Ferro M, Ait Tamlihat Y (2021) Iatrogenic Takotsubo Cardiomyopathy after Accidental Injection of Adrenaline-A Case Report. Ann Case Report 6: 717. DOI: 10.29011/2574-7754.100717

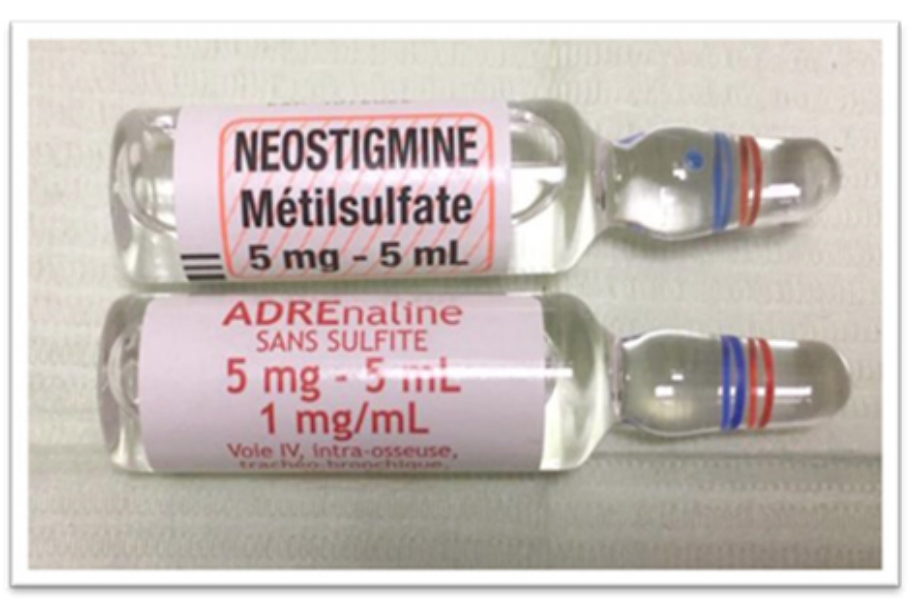

Figure 2: Ampoules NEOSTIGMINE vs ADRENALINE.

Drug administration errors represent a public health problem. Prospective studies show that the error rate in anesthesia is approximately one in 133 anesthesias [9]. In our observation, it was a picking error, the anesthesia nurse took a $5 \mathrm{ml}$ ampoule of epinephrine thinking it was a $5 \mathrm{mg}$ ampoule of prostigmine. Looking at the ampoules, it can be seen that there is no resemblance (Figure 2). Following this serious adverse event, a quick debriefing was done the same day, as well as a morbidity and mortality review and a declaration form to the regional health agency. An anonymized publication on the anesthesia safety network was also made. The frequent use of emergency drugs in anesthesia exposes the risk of medication errors. This clinical case of iatrogenic TTC following accidental injection of adrenaline illustrates the importance of verifying the identity of the drug and its concentration before any preparation and before any administration.

Acknowledgement: None to declare.

Financial Disclosure or Funding: No funding was secured for this study.

Conflict of Interest: The authors have declared that no competing interests exist.

Informed Consent: Written consent signed by the trusted support person
Authors' contributions: MI took charge of the patient. MI and YA drafted the manuscript. LV, FM and MF carried out the critical analysis of article. All authors read and approved the final manuscript.

Data Availability: The authors declare that data supporting the findings of this study are available within the article.

Ethical approval: This study did not need approval of an ethics committee.

\section{References}

1. Sato H, Tateishi H, Uchida T. (1990) Takotsubo type cardiomyopathy due to multivessel spasm. In: Kodama K, Haze K, Hon M, editors. Clinical aspect of myocardial injury: from ischemia to heart failure. Kagaku Hyoronsha; Tokyo: 1990: 56-64.

2. Ghadri JR, Wittstein IS, Prasad A, Sharkey S, Dote K, et al. (2018) International Expert Consensus Document on Takotsubo Syndrome (Part II): Diagnostic Workup, Outcome, and Management. Eur Heart J. 39: 2047-2062.

3. Templin C, Ghadri JR, Diekmann J, Napp LC, Bataiosu DR, et al. (2015) Clinical features and outcomes of Takotsubo (stress) cardiomyopathy. N Engl J Med 373: 929-938.

4. Roshanzamir S, Showkathali R. (2013) Takotsubo cardiomyopathy a short review. Curr Cardiol Rev 9:191-196.

5. Amariles P. (2011) A comprehensive literature search: drugs as possible triggers of Takotsubo cardiomyopathy. Curr Clin Pharmacol. 6: 1-11.

6. Ghadri JR, Wittstein IS, Prasad A, Sharkey S, Dote K, et al. (2018) International Expert Consensus Document on Takotsubo Syndrome (Part I): Clinical Characteristics, Diagnostic Criteria, and Pathophysiology. Eur Heart J.;39: 2032-2046.

7. Stiermaier T, Eitel C, Desch S, Fuernau G, Schuler G, et al. (2018) Incidence, determinants and prognostic relevance of cardiogenic shock in patients with Takotsubo cardiomyopathy. Eur Heart J Acute Cardiovasc Care. 5: 489-496.

8. Choi JH, Oh ID, Shin E. (2020) Extracorporeal membrane oxygenation for takotsubo cardiomyopathy that developed after mitral valve replacement. Acute Crit Care. 35: 51-55.

9. Glavin RJ. (2010) Drug errors: consequences, mechanisms, and avoidance. Br J Anaesth. 105: 76-82. 